\begin{tabular}{|c|l|}
\hline Title & Strong infrared radiation through passive dispersive wave generation and its control \\
\hline Author(s) & Roy, Samudra; Ghosh, Debashri; Bhadra, Shy amal K.; Saitoh, Kunimasa; Koshiba, Masanori \\
\hline Citation & $\begin{array}{l}\text { A pplied Optics, 50(20), 3475-3481 } \\
\text { https://doi.org/10.1364/A0.50.003475 }\end{array}$ \\
\hline Issue Date & 2011-07-10 \\
\hline Doc URL & http://hdl.handle.net/2115/47061 \\
\hline Rights & ○ 2011 Optical Society of A merica \\
\hline Type & article \\
\hline File Information & A 050-20_3475-3481.pdf \\
\hline
\end{tabular}

Instructions for use 


\title{
Strong infrared radiation through passive dispersive wave generation and its control
}

\author{
Samudra Roy, ${ }^{1}$ Debashri Ghosh, ${ }^{2}$ Shyamal K. Bhadra, ${ }^{2, *}$ \\ Kunimasa Saitoh, ${ }^{1}$ and Masanori Koshiba ${ }^{1}$ \\ ${ }^{1}$ Graduate School of Information Science \& Technology, Hokkaido University, Sapporo 060-0814, Japan \\ ${ }^{2}$ Fiber Optics and Photonics Division, Central Glass and Ceramic Research Institute, \\ Council of Scientific and Industrial Research, 196, Raja S.C Mullick Road, Kolkata 700032, India \\ ${ }^{\star}$ Corresponding author: skbhadra@cgcri.res.in
}

Received 17 February 2011; revised 3 May 2011; accepted 18 May 2011; posted 20 May 2011 (Doc. ID 142865); published 5 July 2011

\begin{abstract}
We observe strong infrared (IR) radiation as a result of passive dispersive wave generation for a realistic microstructured fiber having two zero-dispersion wavelengths. The IR radiation frequency can be suitably controlled by varying the operational wavelength, which falls in the first normal dispersion regime. The amplitude of the radiation can be significantly increased by introducing a suitable amount of chirp in the input pulse. This strong phase-matching radiation can be considered as an alternative solution for the IR laser for different applications. (C) 2011 Optical Society of America
\end{abstract}

OCIS codes: $\quad 060.4005,190.4370,190.5650$.

\section{Introduction}

Dispersive wave (DW) generation is a phasematching (PM) phenomenon [1] that occurs when higher-order soliton (HOS) breaks into its fundamental components due to the fission process [2] and transfers energy to the DWs in the form of radiation (sometimes called nonsolitonic radiation [NSR]). Soliton formation in the anomalous dispersion domain is a primary condition to activate such PM resonant radiations. However, a DW may be generated for a specially designed dispersion profile even under pumping in the normal group velocity dispersion (GVD) regime. The formation of such radiation is to some extent unorthodox, and to distinguish such radiation from the conventional process of DW generation, we denote it as passive DW.

We propose a realistic structure of a photonic crystal fiber (PCF) having a defect core that exhibits passive DW when an optical pulse is launched at a normal GVD regime. For such a structure, strong infrared (IR) radiation is obtained, which can be tuned

0003-6935/11/203475-07\$15.00/0

(C) 2011 Optical Society of America by tailoring the pumping wavelength. For different pumping wavelengths, the PM condition of the radiation changes and we have a different radiation frequency each time. A suitable amount of input chirp proves to be an effective parameter in increasing the radiation amplitude by transferring more energy to the DW. Further, the generation of DW radiation takes place earlier when the input pulse is chirped. In our present study we try to evaluate the underlying physics of the generation of passive $\mathrm{DW}$ in the form of IR radiation and the physical parameters that control it. The IR radiation generated through passive DW may meet the increasing demand of an IR laser and may be useful in medical and some strategic applications.

\section{Defect Core PCF}

Several designs of microstructured fibers are proposed to achieve the two or more zero-dispersion wavelengths (ZDWs) [3,4]. However, efficient control over chromatic dispersion and dispersion slope can easily be achieved by a PCF with a defect core [5]. In such fibers, an air hole of submicrometer diameter is placed at the center. For sensing applications, this type of fiber has already been fabricated [6]. The 
design strategy of such fiber is based on the mutual cancellation between the waveguide dispersion and the material dispersion of the PCF. By adjusting the size of the central air hole, it is possible to design ultra-flattened dispersion profile having two zerodispersion (TZD) points. The low confinement loss and high nonlinearity are the additional advantages of such fibers. We consider a PCF with a submicrometer air hole of diameter $\left(d_{c}\right) 0.6 \mu \mathrm{m}$ in the core and air-filling fraction $(f=d / \Lambda)$ of 0.75 [5]. The cladding contains four rings of air holes having diameter $(d)$ $1.5 \mu \mathrm{m}$ with pitch $(\Lambda) 2 \mu \mathrm{m}$. The nonlinearity and leakage loss of fundamental mode (FM) as a function of wavelength is plotted in Fig. 1(a), and the structure of the proposed PCF is shown in the inset. The nonlinear coefficient $(\gamma)$ and leakage loss of FM are calculated to be $18.54 \mathrm{~W}^{-1} \mathrm{~km}^{-1}$ and $0.005 \mathrm{~dB} / \mathrm{km}$, respectively, at the operating wavelength $\left(\lambda_{0}\right)$ of $1550 \mathrm{~nm}$. In the context of the present work, the loss of the fiber at higher wavelengths is critically important. The origination of the losses at higher wavelengths is twofold: first, the characteristic IR absorption of silica material, and second, the leakage loss owing to the finiteness of the periodic air hole structure in the cladding. In Fig. 1(a), we perform our simulation up to $2.4 \mu \mathrm{m}$ for the proposed four air ring defect core PCF. It should be noted that beyond $2.4 \mu \mathrm{m}$, the transmittance of the UV-grade silica (which is generally used in making PCFs) is poor because of the presence of the intensive $\mathrm{OH}$ absorption band in the 2.6-2.8 $\mu \mathrm{m}$ wavelength range. The leakage loss, $L_{c}$, is deduced using the standard formula $L_{c}=8.686 k_{0} \operatorname{Im}\left[n_{\text {eff }}\right]$, where $k_{0}$ is the free space wavenumber and $\operatorname{Im}\left(n_{\text {eff }}\right)$ is the imaginary part of the effective index ( $n_{\text {eff }}$ ) for FM. For the proposed four ring structure, the highest $L_{c}$ is calculated to be $0.16 \mathrm{~dB} / \mathrm{m}$ at the $2.4 \mu \mathrm{m}$ wavelength. This loss can be further reduced by implementing more air holes in the cladding. We examine seven air hole ring structure and the loss is dramatically reduced to $2.5 \times$ $10^{-6} \mathrm{~dB} / \mathrm{m}$ at $2.4 \mu \mathrm{m}$. The transmittance beyond $2.4 \mu \mathrm{m}$ can be improved significantly by using expensive IR-grade silica, which has no $\mathrm{OH}$ absorption band and transmits light up to the $3.5 \mu \mathrm{m}$ wavelength. A major concern at this IR domain is the leakage loss, which increases exponentially with wavelength. An addition of air hole rings in the cladding significantly reduces this leakage loss at higher wavelengths. In Fig. 1(b), we try to compare the leakage loss of a four ring and a seven ring defect core $\mathrm{PCF}$ at the wavelength range 2.5 to $3 \mu \mathrm{m}$ and observe a dramatic improvement for higher air ring structure. It should be mentioned that further addition of air holes influences the dispersion property negligibly since the dispersion profile is primarily controlled by the first two air hole rings.

The dispersion property of the proposed four ring PCF is calculated numerically and it possesses TZD wavelengths at 1531 and $1760 \mathrm{~nm}$, as shown in Fig. 2. The dispersion profile is calculated using the formula $\beta_{2}(\lambda)=\left(\lambda^{3} / 2 \pi c^{2}\right)\left(d^{2} \operatorname{Re}\left[n_{\mathrm{eff}}\right] / d \lambda^{2}\right)$, where $c$ is the ve-

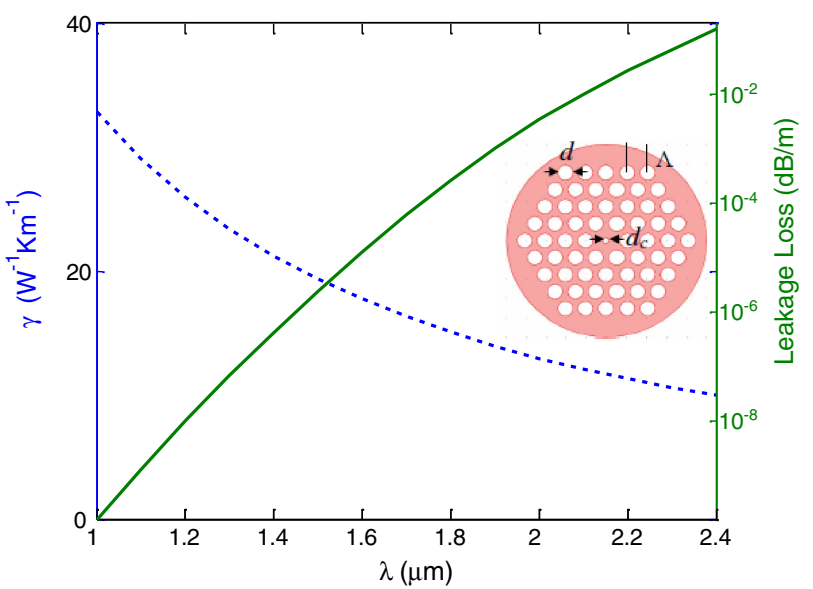

(a)

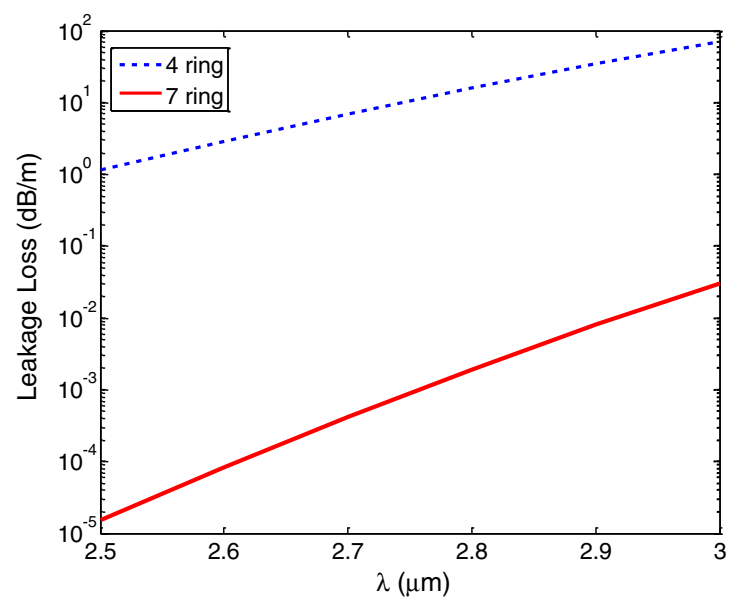

(b)

Fig. 1. (Color online) (a) Variation of nonlinear coefficient $(\gamma)$ (blue dashed curve) and leakage loss (green solid curve) of FM over wavelength is shown for the proposed defect core PCF (inset). (b) Comparison of the leakage loss for four ring and seven ring structures.

locity of the light. The real part of $n_{\text {eff }}\left(\operatorname{Re}\left[n_{\text {eff }}\right]\right)$ at different wavelengths is evaluated using the commercial software COMSOL Multiphysics which is based on finite element method. In the inset of the Fig. 2 the field distribution of the FM is shown for a wavelength of $1520 \mathrm{~nm}$.

\section{Numerical Details}

In order to capture spectral broadening and the effect of higher-order dispersion (HOD) on the propagating pulse under realistic conditions, we employ a generalized nonlinear Schrödinger equation (GNLSE) written in a normalized form as $[\underline{7}, \underline{8}]$

$$
\begin{aligned}
\frac{\partial U}{\partial \xi}= & \frac{i}{2} \frac{\partial^{2} U}{\partial \tau^{2}}+\sum_{m \geq 3}^{\infty} i^{m+1} \delta_{m} \frac{\partial^{m} U}{\partial \tau^{m}}+i N^{2}\left(1+i s \frac{\partial}{\partial \tau}\right) \\
& \times\left(U(\xi, \tau) \int_{-\alpha}^{\tau} R\left(\tau-\tau^{\prime}\right)\left|U\left(\xi, \tau^{\prime}\right)\right|^{2} d \tau^{\prime}\right),
\end{aligned}
$$




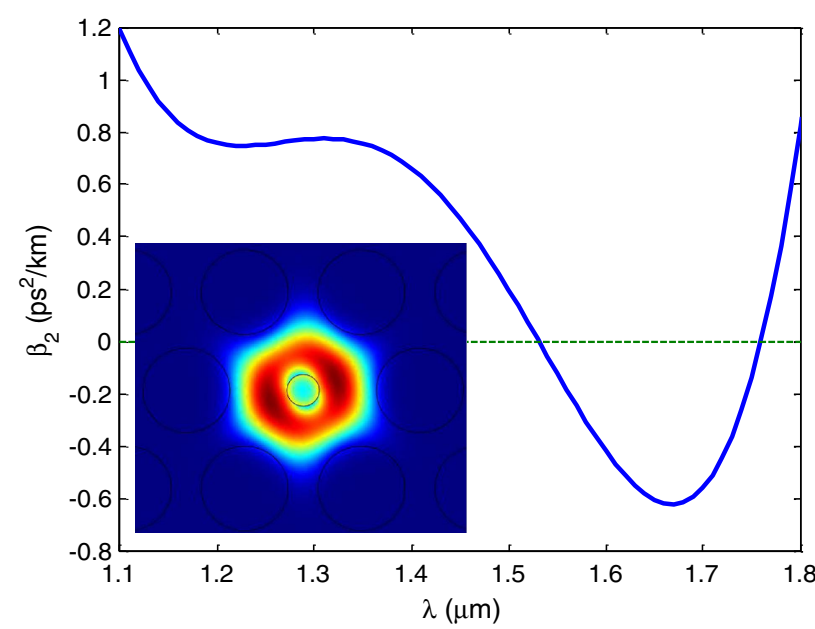

Fig. 2. (Color online) Dispersion profile of the proposed defect core PCF. Inset shows the fundamental field distribution at $1520 \mathrm{~nm}$.

where the field amplitude $U(\xi, \tau)$ is normalized such that $U(0,0)=1$ and the other dimensionless variables are $\xi=\frac{z}{L_{D}}, \tau=\frac{t-z / v_{g}}{T_{0}}, N=\sqrt{\gamma P_{0} L_{D}}$, and $\delta_{m}=\frac{\beta_{m}}{m ! T_{0}^{m-2}\left|\beta_{2}\right|}$.

Here, $N$ defines soliton order, $P_{0}$ is the peak power of the ultrashort pulse launched into the fiber, $T_{0}$ is the input pulse width, $\beta_{m}$ is the $m$ th-order dispersion coefficient in real units, $L_{D}=T_{0}^{2} /\left|\beta_{2}\right|$ is the dispersion length, $\gamma$ is the nonlinear parameter of the fiber, $\delta_{m}$ is the $m$ th-order dispersion coefficient in normalized form, and $s=\left(2 \pi \nu_{0} T_{0}\right)^{-1}$ is the self-steepening parameter at the carrier frequency $\nu_{0}$ of the pulse. The nonlinear response function of the optical fiber has the form [9]

$$
R(\tau)=\left(1-f_{R}\right) \delta(\tau)+f_{R} h_{R}(\tau),
$$

where the first and the second terms correspond to the electronic and Raman responses, respectively, with $f_{R}=0.245$. As discussed in [9], the Raman response function can be expressed in the following form:

$$
h_{R}(\tau)=\left(f_{a}+f_{c}\right) h_{a}(\tau)+f_{b} h_{b}(\tau),
$$

where the functions $h_{a}(\tau)$ and $h_{b}(\tau)$ are defined as

$$
\begin{aligned}
& h_{a}(\tau)=\frac{\tau_{1}^{2}+\tau_{2}^{2}}{\tau_{1} \tau_{2}^{2}} \exp \left(-\frac{\tau}{\tau_{2}}\right) \sin \left(\frac{\tau}{\tau_{1}}\right), \\
& h_{b}(\tau)=\left(\frac{2 \tau_{b}-\tau}{\tau_{b}^{2}}\right) \exp \left(-\frac{\tau}{\tau_{b}}\right)
\end{aligned}
$$

and the coefficients $f_{a}=0.75, f_{b}=0.21$, and $f_{c}=$ 0.04 quantify the relative contributions of the isotropic and anisotropic parts of the Raman response. In Eq. (4), $\tau_{1}, \tau_{2}$, and $\tau_{b}$ have values of 12,32 , and $96 \mathrm{fs}$, respectively. In our notation, they are normalized by the input pulse width $T_{0}$, which is 50 fs for our simulation. Equation (1) is numerically solved by using the split-step Fourier method [7] for a given input pulse having the form $U(0, t)=\operatorname{sech}\left(t / T_{0}\right)$.

\section{Results and Discussions}

It is already mentioned that DW generation is a PM phenomenon and strongly depends on the dispersive property of the waveguide. In the present case, we launch the optical pulse at a wavelength of $1520 \mathrm{~nm}$, which is very close to the first zero-dispersion point $(1531 \mathrm{~nm})$ but lies on the normal dispersion regime of the proposed PCF structure. Since the pulse is launched in the normal dispersion domain, optical soliton ceases to exist. However, because of the nonlinear spectral broadening and Raman frequency downshifting, the pulse immediately enters the intermediate anomalous dispersion domain and eventually forms optical soliton. The evolution of the pulse spectrum is depicted in the spectrogram shown in Fig. 3, where the formation of soliton and the DW around $2027 \mathrm{~nm}$ is clearly identified. The simulation is done for soliton order $N=6$, but the phenomenon can still be generated for $N=5$ or 7 . We make the soliton order high to ensure that sufficient amounts of energy fall in the intermediate anomalous dispersion domain to generate optical soliton and corresponding PM DW. It is examined that for lower soliton orders, for example $N=2$, no $\mathrm{DW}$ is generated in the IR domain. It is evident from Fig. 3 that the Raman-induced frequency downshifting is restricted because of the formation of recoiling radiation at $1925 \mathrm{~nm}$ across the second ZDW (at $1760 \mathrm{~nm}$ ) having a negative dispersion slope [10].

Normally a DW can not be phase-matched with a fundamental soliton whose wavenumber lies in a range forbidden for a linear DW. However, the presence of HODs can lead to phase-matched situations in which energy is transferred from the soliton to DW

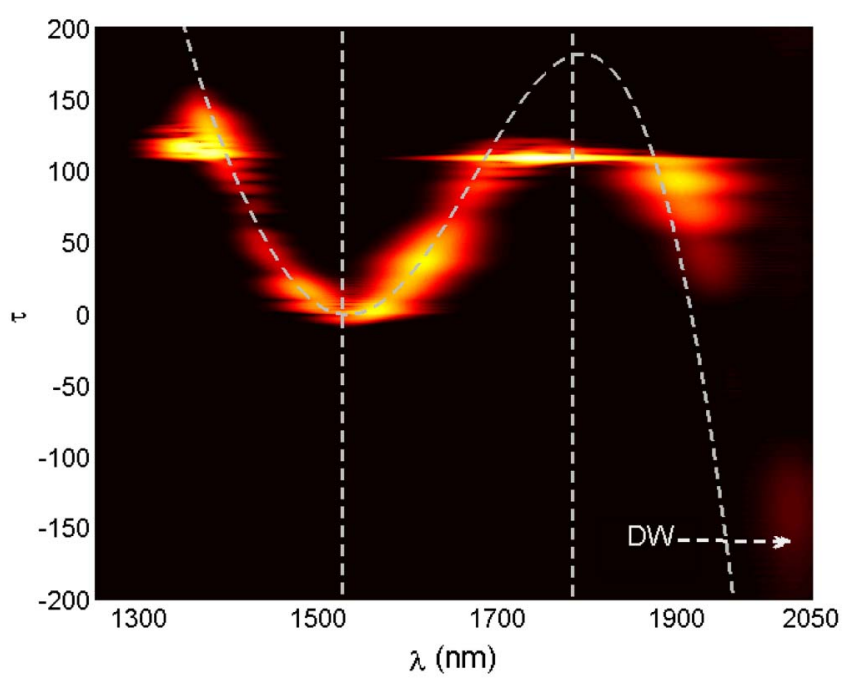

Fig. 3. (Color online) Spectrogram is plotted at normalized propagation distance $\xi=3.5$ for a sixth-order soliton that is launched at the wavelength of $1520 \mathrm{~nm}$. The recoiling radiation appears at roughly $1925 \mathrm{~nm}$ and the DW generates at $2027 \mathrm{~nm}$, as indicated in the figure. The two vertical white dotted lines represent the ZDWs and the curved dotted line corresponds to the group delay. 
at a specific frequency [1,7]. The frequency of the NSR is governed by a simple PM condition which can be given as [7]

$$
\sum_{m=2}^{\infty} \frac{\beta_{m}\left(\omega_{s}\right)}{m !}\left(\omega_{d}-\omega_{s}\right)^{m}=\frac{1}{2} \gamma P_{s}
$$

where $\omega_{s}$ and $\omega_{d}$ are the frequencies of the soliton and the DW, respectively. The different order of dispersion is indicated by the integer $m . P_{s}$ is peak power of the Raman soliton formed after the fission process, and $\gamma$ is the nonlinear coefficient already defined.

It is already mentioned that the generation of IR radiation as a DW is a nonconventional process since the pumping wavelength falls in the normal GVD domain. The existing theory [8] and the experiment [11] suggest that a TZD profile always exhibits dual radiation at anomalous GVD pumpings where fourth-order dispersion plays a significant role. The spectrogram in Fig. 3 also predicts dual radiation, one around $2027 \mathrm{~nm}$ and another around $1400 \mathrm{~nm}$. In the present case, we mainly concentrate on the radiation that takes place in the IR regime $(2027 \mathrm{~nm})$ and try to find out the parameters to control it. As shown in Fig. 4, under normal GVD pumping, a strong IR radiation is generated as a DW whose wavelength can be controlled by varying the wavelength $\left(\lambda_{0}\right)$ of the input pulse. In our simulation, pumping wavelength is varied over $120 \mathrm{~nm}$ (from 1400 to $1520 \mathrm{~nm}$ ) and we obtain a wide range of IR radiation $\left(\lambda_{R}\right)$ covering $1500 \mathrm{~nm}$ (from 2000 to $3500 \mathrm{~nm}$ ). This unique feature is only observed when we pump the pulse in the normal GVD domain to allow the generation of passive DWs. However, it is also observed that in anomalous GVD pumpings, we achieve radiations that are mediated by HOS but are within the shorter IR wavelength (less than $2000 \mathrm{~nm}$ ) region.

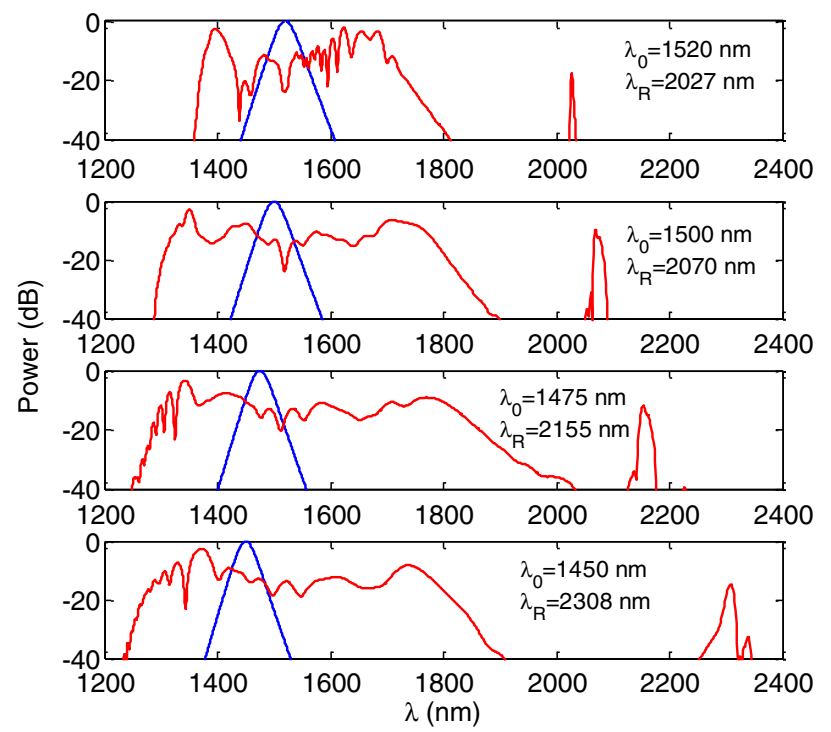

Fig. 4. (Color online) Generation of strong IR radiation as a passive DW at one normalized distance $(\xi=1)$ for different operating wavelengths. The soliton order is $N=6$.
Figure 4 qualitatively describes that radiation wavelength and amplitude significantly depend on the operating wavelength. In order to visualize this dependence more clearly, in Fig. $\underline{5}$ we plot the frequency and amplitude of the IR radiation simultaneously as a function of different pump wavelengths, which reveals interesting facts. The red diamonds with black dotted curve represent the wavelengths of radiations that are obtained by solving the conventional PM condition as given in Eq. (5), whereas the blue circles give the simulated data that we get by solving the GNLSE. It may be noted that the two results are close but do not coincide accurately. The possible reason of this mismatch may be understood by the following explanation. The PM condition is basically a polynomial whose coefficients depend on the numeric values of the HOD coefficient at soliton frequency. In the present case, we obtain the solutions of Eq. (5) in terms of radiation wavelengths, by putting the HOD values at operating wavelengths which fall in the normal GVD domain where soliton is not formed. In fact, soliton is generated when the pulse enters the anomalous dispersion domain because of Raman frequency downshifting after a certain propagation distance. DW radiation takes place physically under this condition. Hence, the values of HOD coefficients used in the expression are incorrect in the sense that optical soliton is yet to be generated at slightly different wavelengths to form any DW radiation. However, it is quite difficult to identify the precise location where optical soliton begins to generate during the process and the corresponding HOD coefficients at that specific wavelength. Amplitude of the IR radiation is another important issue from the application point of view. In Fig. 5, we also plot the amplitudes of IR radiation generated at different pumping wavelengths at $\xi=10$. The result shows that the amplitudes are almost stable and gradually improving when the pump wavelength is set closer to the first zero-dispersion point except for the case $\lambda_{0}=1520 \mathrm{~nm}$. An abrupt drop of the amplitude is noticed when the pumping wavelength $(1520 \mathrm{~nm})$ is too close to the first ZDW $(1531 \mathrm{~nm})$. For pumping at $1520 \mathrm{~nm}$, which is too close to the first ZDW, the spectrum encounters the second ZDW at a relatively shorter distance due to Raman frequency downshifting and radiates strongly. Under such process, as shown in Fig. 3, most of the pulse energy is transferred to the recoiling radiation at $1925 \mathrm{~nm}$ across the second ZDW where the dispersion slope is negative. This energy transformation affects the IR radiation and eventually reduces the amplitude of the DW at $2027 \mathrm{~nm}$. For other wavelengths, however, the spectral recoiling effects take place at later distances, which results in relatively stronger DW radiations in the IR domain. Moreover, the amplitude of the third-order dispersion coefficient $\left(\delta_{3}\right)$ increases for 1400 to $1520 \mathrm{~nm}$, which is also a reason for stronger DW radiations $[\underline{1}, \underline{12}]$. 


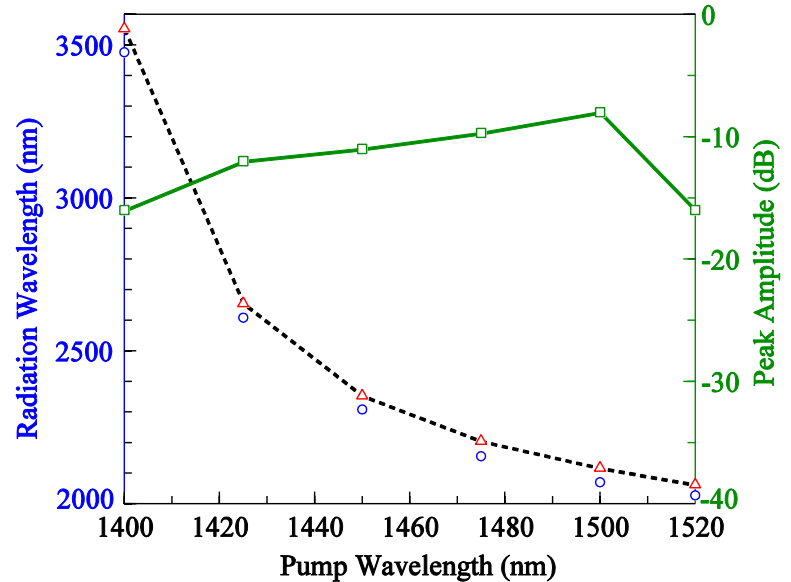

Fig. 5. (Color online) Variation of the radiation wavelength with pump wavelength. The blue circles represent the simulated data and the red triangles show the data obtained from the PM condition (Eq. (5)). The green squares represent the peak amplitudes of the corresponding DWs at $\xi=10$.

We further quantitatively investigate the generation of appreciable DW over fiber distance. In order to do that, we consider the threshold peak power of IR radiation to be $-20 \mathrm{~dB}$. Now we define a critical length $\left(\xi_{20 \mathrm{~dB}}\right)$ at which the peak power of the IR radiation reaches $-20 \mathrm{~dB}$. In Fig. 6 , we plot $\xi_{20 \mathrm{~dB}}$ for different operating wavelengths and find a general tendency that DW generates at longer distance when the operating wavelengths move away from the first ZDW, which is at $1531 \mathrm{~nm}$. However, for the operating wavelength of $1520 \mathrm{~nm}$, the peak power of DW reaches $-20 \mathrm{~dB}$ at a relatively longer distance because of the energy transfer to the recoiling radiation that is generated across the second ZDW at $1925 \mathrm{~nm}$ (Fig. 1) as a result of the cancellation of Raman frequency downshifting [10].

In Fig. 6, we simultaneously plot the critical normalized distance in actual units $\left(z=\xi_{20 \mathrm{~dB}} \times L_{D}\right)$ and it also reveals that, for the given fiber structure,

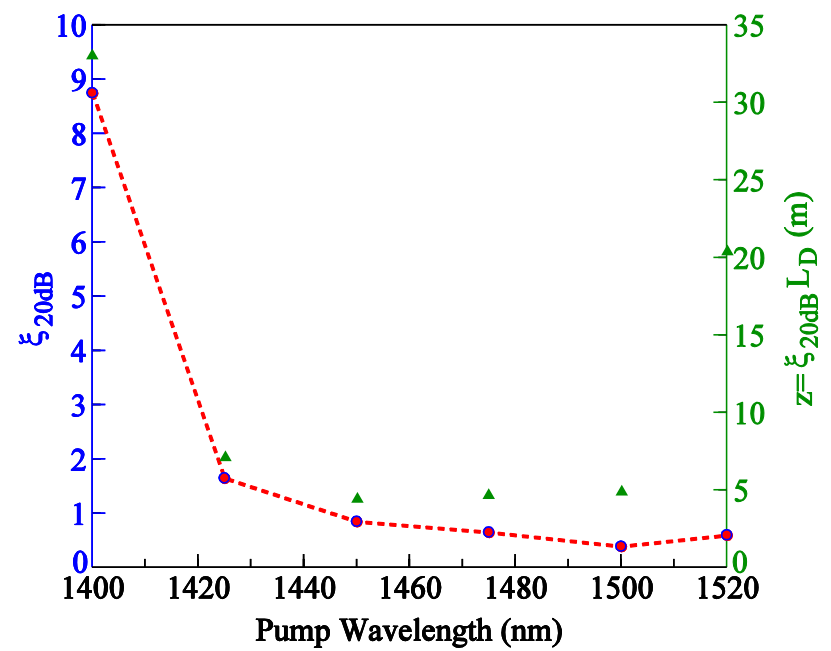

Fig. 6. (Color online) Critical normalized distance $\left(\xi_{20 \mathrm{~dB}}\right)$ as a function of operating wavelengths is given by the red dots whereas the corresponding values in actual units are depicted as green triangles. appreciable DW radiation (with $-20 \mathrm{~dB}$ peak power level) in the IR domain can be achieved within $7.5 \mathrm{~m}$ of fiber length at operating wavelengths of 1425 to $1500 \mathrm{~nm}$, whereas for $\lambda_{0}=1400$ and $1520 \mathrm{~nm}$, it generates beyond $20 \mathrm{~m}$. At this point, it is pertinent to mention that the characteristic IR absorption of UV-grade silica should produce detrimental effects on the conversion efficiency of the radiations beyond the $2.5 \mu \mathrm{m}$ wavelength. However, the radiations that are less than $2.4 \mu \mathrm{m}$ (as shown in Fig. 4) are well within the transmission window of UV-grade silica and, hence, experience relatively less absorption loss. The use of expensive IR-grade silica in PCF, however, may remove the limitation of light transmission beyond $2.5 \mu \mathrm{m}$.

\section{Role of Input Chirp}

In the present work, our aim is to generate moderate IR radiation in the form of phase-matched DW and to control it. So far, it is shown that the radiation may be tuned suitably by changing the operating wavelength. The peak amplitude is also calculated for different pumping wavelengths. In this part of the study, we try to reveal the role of input chirp in enhancing the DW radiation. It is known that optical chirp influences the recoiling radiation and DW dramatically [13,14]. In Fig. 6, it is already shown how critical length $\bar{\xi}_{20 \mathrm{~dB}}$, which is basically a measure of fiber length at which we may have appreciable DW radiation (peak power of $-20 \mathrm{~dB}$ ), varies with operating wavelength. According to that study, more than $7 \mathrm{~m}$ of defect core PCF is required to generate appreciable IR radiation. However, it can be shown that with suitable input chirp, not only the peak power of the radiation is improved, but the critical length is significantly reduced. If we can reduce the critical length, then we need a smaller length of fiber for generating IR radiation and the loss will minimize. For a chirped pulse, the input field is in the form $U(0, \tau)=$ $\operatorname{sech}(\tau) \exp \left(-i C \tau^{2}\right), C$ being the normalized chirping parameter. In Fig. 7 , we try to show how, for a chirped input pulse, we can improve the amplitude of the radiation. In Fig. 7, we enlarge the radiation part of the spectrum and plot it at a distance for which the peak amplitude (red middle curve) reaches $-20 \mathrm{~dB}$ when input pulse is chirped as $C=0.5$. To compare with an unchirped condition, we also plot the DW radiation at same distance in the blue (lower) curve for $C=0$. It is also shown that if we increase the input chirp to $C=1$, then we have even better results at the same distance, as shown by black (upper) curve. It is calculated that the critical length $\left(\xi_{20 \mathrm{~dB}}\right)$ reduces from 4.51 to $3.97 \mathrm{~m}$ for $\lambda_{0}=1450 \mathrm{~nm}, 4.72$ to $3.48 \mathrm{~m}$ for $\lambda_{0}=1475 \mathrm{~nm}, 4.91$ to $4.21 \mathrm{~m}$ for $\lambda_{0}=1500 \mathrm{~nm}$, and finally from 20.09 to $11.75 \mathrm{~m}$ for $\lambda_{0}=1520 \mathrm{~nm}$ when the input chirp value is increased from $C=0$ to $C=0.5$. The respective distances further reduce to $3.28 \mathrm{~m}$ for $\lambda_{0}=1450 \mathrm{~nm}, 2.98 \mathrm{~m}$ for $\lambda_{0}=1475 \mathrm{~nm}$, $3.33 \mathrm{~m}$ for $\lambda_{0}=1500 \mathrm{~nm}$, and $5 \mathrm{~m}$ for $\lambda_{0}=1520 \mathrm{~nm}$ when we increase the chirp to 1 . Clearly, the input chirp becomes most effective when the operating 

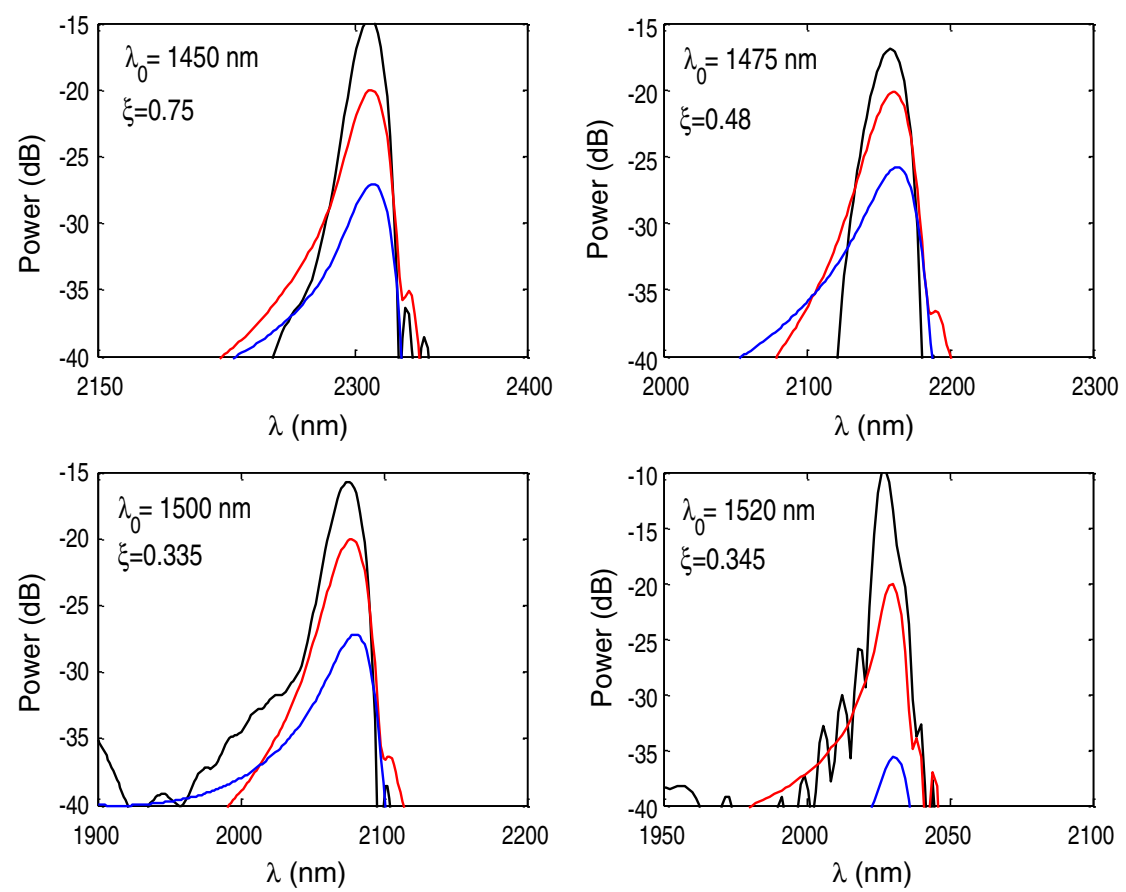

Fig. 7. (Color online) Improvement of the peak amplitude of DW radiation is shown for three different chirp values at four different operating wavelengths. The blue (lower), red (middle), and black (upper) curves represent the input chirp $C=0,0.5$, and 1 , respectively. In all four cases, the spectra is plotted at a distance for which the radiation peak reaches $-20 \mathrm{~dB}$ for the input pulse having chirp $C=0.5$.

wavelength is $1520 \mathrm{~nm}$ and appreciable radiation can be obtained well within $5 \mathrm{~m}$ of PCF. It is to be mentioned that DWs are generated in the IR domain where the silica has a significant absorption and leakage loss. We can minimize the effect of loss by generating DWs at shorter distances. We further try to study the evolution of DW peak over distance and obtain the curves as shown in Fig. 8(a). Two different cases are considered, one in which the input pulse is unchirped (blue lower curve) and another where the input pulses are chirped $(C=0.5$ and 1$)$ for $\lambda_{0}=1520 \mathrm{~nm}$. From Fig. 8(a), it is clear that a suitable amount of input chirp enhances the radiation power significantly. Most importantly, the amplification of the radiation peak takes place at shorter distances when the input pulse is chirped. In the present case, $\xi_{20 \mathrm{~dB}}$ reduces from $0.59(20.09 \mathrm{~m})$ to $0.345(11.75 \mathrm{~m})$ when the input chirp is increased from 0 to 0.5 . The distance is further reduced to $0.147(5 \mathrm{~m})$ when the input chirp is increased to 1 . For the $1520 \mathrm{~nm}$ input, the radiation appears at the wavelength of $2027 \mathrm{~nm}$. The leakage loss at that wavelength is calculated to be less than $0.007 \mathrm{~dB} / \mathrm{m}$, which is well within permissible limits. The most redshifted DW radiation, within the transmission window limit of $2.4 \mu \mathrm{m}$, appears when we pump at the wavelength of $1450 \mathrm{~nm}$. The corresponding DW radiation evolves at around $2300 \mathrm{~nm}$, where the leakage loss is calculated to be $0.065 \mathrm{~dB} / \mathrm{m}$. At this wavelength, less than $4 \mathrm{~m}$ of fiber is required to produce appreciable DW; hence, the maximum accumulated loss should be less than $0.3 \mathrm{~dB}$, which is a legitimate amount in the context of practical application. However, this loss can be dramatically reduced to
$3.68 \times 10^{-7} \mathrm{~dB} / \mathrm{m}$ for the seven ring structure, and we have practically zero leakage loss for this radiation.

It should be noted that in all the cases, the growth of peak amplitude saturates over the distance. Most importantly, the saturated peak power of the DW radiation improves marginally when we increase the chirp value from 0.5 to 1 . We also calculate the percentage of energy transfer to the DW as defined by $\left(E_{\mathrm{DW}} / E_{\text {Total }}\right) \times 100 \%$, with $E_{\mathrm{DW}}$ and $E_{\text {Total }}$ being the energy of DW radiation and total spectrum, respectively. In Fig. 8(b), we plot the energy transfer as a function of propagation length for three different chirp values at $\lambda_{0}=1520 \mathrm{~nm}$. It is evident from Fig. 8(b) that the energy transfer to the DW is significantly improved for chirped pulses. At this point, it is relevant to mention that the peak amplitude of the DW as well as the energy conversion efficiency will not increase indefinitely with increasing chirp but will saturate. Since the optical pulse is launched in the normal dispersion domain, most of the pulse energy initially refrains from generating any optical soliton. However, during propagation, the pulse experiences nonlinear spectral broadening and Raman frequency downshifting. Because of this redshifting, some parts of the pulse energy now enter in the anomalous dispersion domain and eventually form optical soliton. At this point, it should be remembered that since a section of the entire pulse energy enters the anomalous dispersion domain and forms optical soliton, the effective soliton order should be less than what is originally used in the input. Hence, in this case the effective soliton order is less than 6 . For a chirped pulse, on the other hand, the broadening accelerates and more energy is allowed to enter 


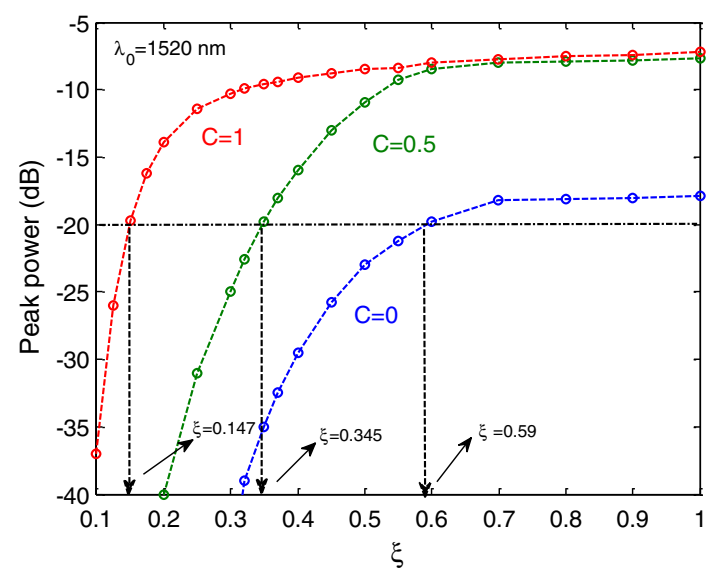

(a)

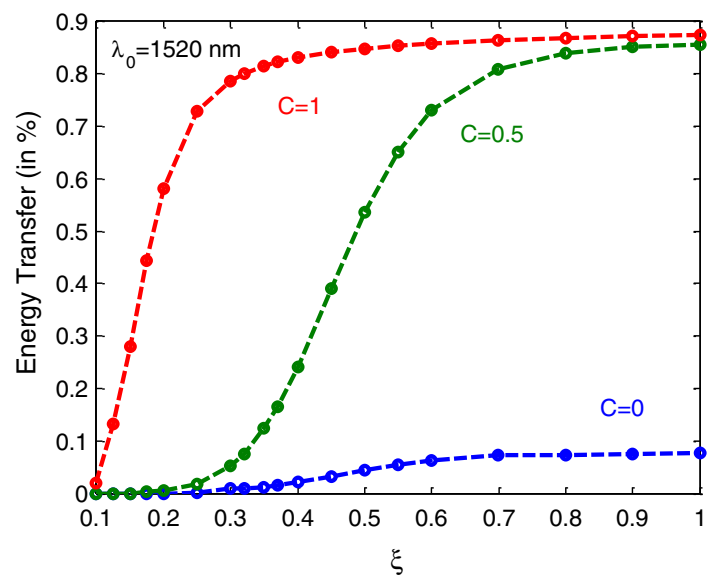

(b)

Fig. 8. (Color online) (a) Growth of the peak amplitude of the DW radiation is shown over distance for three different chirp values $C=0$ (blue lower curve), 0.5 (green middle curve), and 1 (red upper curve), at operating wavelengths of $1520 \mathrm{~nm}$ with soliton order $N=6$. (b) Energy transfer is plotted as a function of propagation distance for the same set of parameters.

in the anomalous dispersion domain to form optical solitons. In this case, the effective soliton order is increased, and because of this, DW generates at shorter distances with increasing amplitudes. However, the effective soliton order cannot be increased indefinitely with the increment of input chirp, and we observe a saturation of the conversion efficiency that is consistent with the result presented in [12]. Since it is difficult to generate laser light at any desirable wavelength, the efficient transfer of energy to new tunable wavelengths and its control are of great importance from an application point of view. The use of defect core $\mathrm{PCF}$ in generating passive $\mathrm{DW}$ may prove to be an attractive solution to generating convenient and efficient IR lasers in the near future for a wide range of applications.

\section{Conclusion}

It is demonstrated that efficient IR radiation can be generated under normal GVD pumping in the form of passive DW. A defect core PCF is proposed that exhibits strong resonant radiations in the IR domain. The radiation wavelength is found to be quite sensitive with input pump wavelength and can be tailored suitably. The growth of such radiation is described quantitatively. It is demonstrated that a suitable amount of input chirp can significantly improve the radiation amplitude and the corresponding energy transfer. Structural modification of the proposed PCF reduces the exponential increment of leakage loss in the IR domain. It is concluded that such strong IR radiation can only be possible for passive DW generation and could be an alternative solution for tunable IR laser in medical and strategic applications.

\section{References}

1. N. Akhmediev and M. Karlsson, "Cherenkov radiation emitted by solitons in optical fibers," Phys. Rev. A 51, 2602-2607 (1995).

2. A. V. Husakou and J. Herrmann, "Supercontinuum generation of higher order solitons by fission in photonic crystal fibers," Phys. Rev. Lett. 87, 203901 (2001).

3. B. Kibler, P. A. Lacourt, F. Courvoisier, and J. M. Dudley, "Soliton spectral tunneling in photonic crystal fiber with sub-wavelength core defect," Electron. Lett. 43, 967-968 (2007).

4. F. Poletti, P. Horak, and D. J. Richardson, "Soliton spectral tunneling in dispersion controlled holey fibers," IEEE Photon. Technol. Lett. 20, 1414-1416 (2008).

5. K. Saitoh, N. Florous, and M. Koshiba, "Ultra-flattened chromatic dispersion controllability using a defect-core photonic crystal fiber with low confinement losses," Opt. Express 13, 8365-8371 (2005).

6. X. Yu, Y. Sun, G. B. Ren, P. Shum, N. Ngo, and Y. C. Kwok, "Evanescent field absorption sensor using a pure-silica defect-core photonic crystal fiber," IEEE Photon. Technol. Lett. 20, 336-338 (2008).

7. G. P. Agrawal, Nonlinear Fiber Optics, 4th ed. (Academic, 2008).

8. S. Roy, S. K. Bhadra, and G. P. Agrawal, "Effect of higher order dispersion on resonant dispersive wave emitted by solitons," Opt. Lett. 34, 2072-2074 (2009).

9. Q. Lin and G. P. Agrawal, "Raman response function for silica fibers," Opt. Lett. 31, 3086-3088 (2006).

10. D. V. Skryabin, F. Luan, J. C. Knight, and P. St. J. Russell, "Soliton self-frequency shift cancellation in photonic crystal fiber," Science 301, 1705-1708 (2003).

11. F. Benabid, F. Biancalana, P. S. Light, F. Couny, A. Luiten, P. J. Roberts, J. Peng, and A. V. Sokolov, "Fourth-order dispersion mediated solitonic radiations in HC-PCF cladding," Opt. Lett. 33, 2680-2682 (2008).

12. S. Roy, S. K. Bhadra, and G. P. Agrawal, "Dispersive wave emitted by solitons perturbed by third-order dispersion inside optical fibers," Phys. Rev. A 79, 023824 (2009).

13. S. Roy, D. Ghosh, S. K. Bhadra, K. Saitoh, M. Koshiba, and G. P. Agrawal, "Impact of chirp on spectral recoil of solitons in a defect-core photonic fiber with two zero dispersion wavelengths," in Optical Fiber Communication Conference, OSA Technical Digest (Optical Society of America, 2011), paperOTuA2.

14. D. Lei, H. Dong, S. Wen, and H. Yang, "Manipulating dispersive wave generation by frequency chirp in photonic crystal fibers," J. Lightwave Technol. 27, 4501-4507 (2009). 